\title{
A NOTE ON EXTENSIONS GENERATED BY CLOSED SETS
}

\author{
Maximilian Ganster
}

\begin{abstract}
A bstract. In a recent paper Abd El-Monsef et al. consider a certain topology on $2^{X}$ where $2^{X}$ is the family of all nonempty closed subsets of a given topological space $X$. Unfortunately, several results in their paper are incorrect and so the purpose of this note is to correct, improve and expand these results. In addition, the main quesion in their paper turns out to have a quite simple answer.
\end{abstract}

\section{Introduction and Preliminaries}

For a topological space $(X, \tau)$ the closure of a subset $A$ of $X$ is denoted by $\operatorname{cl}_{\tau} A$ and we will suppress the $\tau$ when there is no confusion possible. A space $(X, \tau)$ is called $R_{0}$ if for any open set $U$ containing a point $x$ we have $\operatorname{cl}\{x\} \subseteq U$. A subset $S$ of $(X, \tau)$ is called locally closed [2] if $S$ is the intersection of an open set and a closed set, or equivalently, if $S=U \cap \operatorname{cl} S$ for some open set $U$. We will call a subset $S$ of $(X, \tau)$ an $F_{\alpha}$-set if $S$ is the union of closed subsets of $(X, \tau)$.

Observation 1.1 . A space $(X, \tau)$ is $T_{1}$ (resp. $R_{0}$ ) if and only if every subset (resp. every open subset) is an $F_{\alpha}$-set.

No separation axioms are assumed unless stated explicitly. Finally, the set of natural numbers is denoted by $\omega$.

\section{The correspondence $U \rightarrow U^{*}$}

Following Michael [3], for a space $(X, \tau)$ let $2^{X}$ be the family of all nonempty closed subsets of $(X, \tau)$.

Definition. For each open subset $U$ of a space $(X, \tau)$ let $U^{*}=\left\{F \in 2^{X}: F \subseteq U\right\}$.

Received November 9, 1993; revised February 15, 1994.

1991 Mathematics Subject Classification. 54A10, 54D10, 54B20.

Key words and phrases. Extension of spaces, hyperspace. 
We now investigate the correspondence $U \rightarrow U^{*}$ for arbitrary spaces $(X, \tau)$. It turns out that ii), iii) and iv) of Theorem 2.1 in [1] are incorrect and have to be modified.

Observation 2.1. If $U$ and $V$ are open subsets of $(X, \tau)$ then

1) $\varnothing^{*}=\varnothing$ and $X^{*}=2^{X}$,

2) $(U \cap V)^{*}=U^{*} \cap V^{*}$,

3) if $U \subseteq V$ then $U^{*} \subseteq V^{*}$,

4) $U^{*} \cup V^{*} \subseteq(U \cup V)^{*}$.

Consider the following conditions where $U$ and $V$ are arbitrary open sets in $(X, \tau)$ :

(P1) $U^{*} \subseteq V^{*}$ implies $U \subseteq V$,

(P2) $U \neq \varnothing$ implies $U^{*} \neq \varnothing$,

(P3) $(U \cup V)^{*} \subseteq U^{*} \cup V^{*}$.

It turns out that these conditions have quite useful characterizations. First observe that for open subsets $U$ and $V$ of $(X, \tau), U \not \subset V$ if and only if $U-V$ is nonempty and locally closed. In addition, $U^{*} \not \subset V^{*}$ if and only if there is a nonempty closed set contained in $U-V$.

Theorem 2.2. For a space $(X, \tau)$ the following are equivalent:

1) $(X, \tau)$ satisfies $(P 1)$,

2) every nonempty locally closed set $A$ contains an $F_{\alpha}$-set which is dense in $A$.

Proof. 1) $\Rightarrow$ 2) : Let $A \subseteq X$ be locally closed, i.e. $A=U \cap \mathrm{cl} A$ for some open set $U$. If $S=U\{F \subseteq X: F$ is closed and $F \subseteq A\}$ then $S$ is an $F_{\alpha}$-set and $S \subseteq A$. We claim that $A \subseteq \mathrm{cl} S$. Suppose there exists $x \in A$ with $x \notin \mathrm{cl} S$. Then there exists an open set $G$ containing $x$ with $G \subseteq U$ and $G \cap S=\varnothing$. Now pick $F \in G^{*}$. If $F \cap \operatorname{cl} A$ is nonempty then $F \cap \mathrm{cl} A \subseteq U \cap \mathrm{cl} A=A$ and so $F \cap \mathrm{cl} A \subseteq S$, a contradiction. Hence $F \cap \mathrm{cl} A=\varnothing$ and so $G^{*} \subseteq(X-\mathrm{cl} A)^{*}$. By condition (P1) we have $G \subseteq X-\operatorname{cl} A$ and $G \cap A=\varnothing$, a contradiction. Thus $A \subseteq \mathrm{cl} S$ and we are done.

2) $\Rightarrow 1$ ) : Let $U, V \subseteq X$ be open with $U^{*} \subseteq V^{*}$. Suppose that $U \not \subset V$. If $A=U \cap(X-V)$ then $A$ is nonempty and locally closed, so there exists an $F_{\alpha}$-set $S$ with $S \subseteq A \subseteq$ cl $S$. In particular there exists $F \in 2^{X}$ with $F \subseteq S$. Clearly $F \in U^{*}$ and $F \notin V^{*}$, a contradiction. Thus $U \subseteq V$.

Theorem 2.3. For a space $(X, \tau)$ the following are equivalent:

1) $(X, \tau)$ satisfies (P2),

2) every nonempty openetet $U$ contains an $F_{\alpha}$-set $S$ which is dense in $U$.

Proof. 1) $\Rightarrow$ 2) : Let $U \subseteq X$ be open and let $S=\cup\{F \subseteq X: F$ is closed and $F \subseteq U\}$. Then $S$ is an $F_{\alpha}$-set with $S \subseteq U$. We claim that $U \subseteq$ cl $S$. Suppose that $U \cap(X-\mathrm{cl} S)$ is nonempty. By condition (P2) there exists $F \in 2^{X}$ with $F \subseteq U$ and $F \cap S=\varnothing$, a contradiction. Thus $U \subseteq$ cl $S$ and we are done. 
2) $\Rightarrow$ 1) : Let $U \neq \varnothing$ be open. By assumption there exists an $F_{\alpha}$-set $S$ with $S \subseteq U \subseteq \operatorname{cl} S$. In particular there exists $F \in 2^{X}$ with $F \subseteq S$ and so $F \in U^{*}$, i.e. $U^{*} \neq \varnothing$.

Theorem 2.4. For a space $(X, \tau)$ the following are equivalent:

1) $(X, \tau)$ satisfies $(P 3)$,

2) if $F_{1}, F_{2} \in 2^{X}$ then $F_{1} \cap F_{2} \neq \varnothing$,

3) if $U \neq X$ is open then $U^{*}=\varnothing$.

Proof. 1) $\Rightarrow 2$ ): Let $F_{1}, F_{2} \in 2^{X}$ and suppose that $F_{1} \cap F_{2}=\varnothing$. If $U=$ $X-F_{1}, V=X-F_{2}$ and $F=F_{1} \cup F_{2}$ then $U$ and $V$ are open with $U \cup V=X$ and so $F \in(U \cup V)^{*}$, i.e. $F \subseteq X-F_{1}$ or $F \subseteq X-F_{2}$, a contradiction. Hence $F_{1} \cap F_{2} \neq \varnothing$.

2) $\Rightarrow 3$ ) : Let $U \neq X$ be open and suppose there exists $F \in U^{*}$. Since $X-U$ is nonempty and closed, we have $F \cap(X-U)=\varnothing$, a contradiction. Thus $U^{*}=\varnothing$.

$3) \Rightarrow 2)$ : Let $F_{1}, F_{2} \in 2^{X}$. By assumption $\left(X-F_{1}\right)^{*}=\varnothing$ and so $F_{2} \notin\left(X-F_{1}\right)^{*}$, i.e. $F_{1} \cap F_{2} \neq \varnothing$.

2) $\Rightarrow 1):$ Let $U, V \subseteq X$ be open and let $F \in(U \cup V)^{*}$. Suppose that $F \notin U^{*} \cup V^{*}$. If $F_{1}=F \cap(X-U)$ and $F_{2}=F \cap(X-V)$ then $F_{1}, F_{2} \in 2^{X}$. By assumption $F_{1} \cap F_{2} \neq \varnothing$, a contradiction. Thus $F \in U^{*} \cup V^{*}$.

Corollary 2.5. If $(X, \tau)$ is $T_{1}$ and $|X| \geq 2$ then $(X, \tau)$ does not satisfy $(P 3)$.

From the previous results it is clear that for a space $(X, \tau)$ the following implications hold:

$$
T_{1} \Rightarrow R_{0} \Rightarrow(P 1) \Rightarrow(P 2)
$$

We will now point out that none of these implications is reversible and that there are spaces which do not satisfy (P2). First note that the indisctete topology on an infinite set yields an $R_{0}$ space which is not $T_{1}$.

Example 2.6. Let $X$ be an infinite set and let $p \in X$. It is clear that $\tau=$ $\{\varnothing\} \cup\{G \subseteq X: p \in G$ and $X-G$ is finite $\}$ is a topology on $X$. Note that $\{x\}$ is closed whenever $x \neq p$ and that $\{p\}$ is dense. Hence $(X, \tau)$ is not $R_{0}$. We now show that $(X, \tau)$ satisfies (P1). Let $U, V \subseteq X$ be open with $U^{*} \subseteq V^{*}$ and let $x \in U$. If $x \neq p$ then $\{x\} \in U^{*}$ and so $x \in V$. If $x=p$ then there exists $y \neq p$ with $y \in U$, and so $\{y\} \in U^{*}$, hence $V \neq \varnothing$ and $p \in V$. Thus $U \subseteq V$.

Example 2.7. Let $R$ denote the set of reals and let $X=R \cup\{p\}$ where $p \notin R$. $A$ topology $\tau$ on $X$ is defined as follows. Basic neighbourhoods of $x \in R$ are of the form $(x-\varepsilon, x+\varepsilon)$ where $\varepsilon>0$. A basic neighbourhood of $p$ is of the form $\{p\} \cup(-\varepsilon, \varepsilon)$ with $\varepsilon>0$. It is easy to check that $(X, \tau)$ satisfies (P2) (in fact, every open set contains a suitable $\tau$-closed interval $[a, b])$. If $F \subseteq R$ is nonempty and closed then $p \notin F$ and thus $0 \notin F$. So $R^{*} \subseteq(R-\{0\})^{*}$ but obviously $R$ is not contained in $R-\{0\}$, i.e. $(X, \tau)$ does not satisfy (P1). 
Example 2.8. Let $\tau$ be the following topology on $\omega, \tau=\{\varnothing\} \cup\{1, \ldots, n\}: n \in \omega\}$. By Theorem 2.4. it is clear that $(\omega, \tau)$ satisfies (P3). Obviously $(\omega, \tau)$ does not satisfy (P2).

\section{The Space $\left(2^{X}, \sigma\right)$}

It is well known that, given a space $(X, \tau)$, one may define several quite interesting topologies on $2^{X}$, see e.g. [3], In [4] Schmidt discusses a certain topology $\sigma$ on $2^{X}$ which has already been mentioned by Michael in [3]. In their recent paper Abd El-Monsef et al. [1] continued the study of this space $\left(2^{X}, \sigma\right)$ and we will now improve and correct some of their results.

Given a space $(X, \tau)$ it is clear from Observation 2.1. that $\left\{U^{*}: U \subseteq X\right.$ is open in $(X, \tau)\}$ is a base for a topology $\sigma$ on $2^{X}$. This topology $\sigma$ will be considered throughout this section.

Theorem 3.1. For a space $(X, \tau)$ let $F_{1}, F_{2} \in 2^{X}$. If $F_{1} \subseteq F_{2}$ then $F_{2} \in$ $c l_{\sigma}\left\{F_{1}\right\}$. If $(X, \tau)$ is $R_{0}$ then the converse is also true.

Proof. Suppose that $F_{2} \notin \mathrm{cl}_{\sigma}\left\{F_{1}\right\}$. Then there exists $U \subseteq X$ open in $(X, \tau)$ with $F_{2} \in U^{*}$ and $U^{*} \cap\left\{F_{1}\right\}=\varnothing$, i.e. $F_{1} \notin U^{*}$ and this is a contradiction.

Now let $(X, \tau)$ be $R_{0}$ and let $F_{2} \in \operatorname{cl}_{\sigma}\left\{F_{1}\right\}$. Suppose that there exists $x \in F_{1}-F_{2}$. Then $\operatorname{cl}_{\tau}\{x\} \subseteq X-F_{2}$ and so $F_{2} \in\left(X-\operatorname{cl}_{\tau}\{x\}\right)^{*}$. By hypothesis $F_{1} \in\left(X-\operatorname{cl}_{\tau}\{x\}\right)^{*}$ and consequently $x \notin F_{1}$, a contradiction. Hence $F_{1} \subseteq F_{2}$.

Corollary 3.2. If $(X, \tau)$ is $T_{1}$ and $|X|>1$ then $\left(2^{X}, \sigma\right)$ is not $T_{1}$. Hence Theorem 3.1., Theorem 4.1., Theorem 4.2., and Theorem 4.3. in [1] are false.

Corollary 3.3. Let $W \subseteq 2^{X}$ be an open set in $\left(2^{X}, \sigma\right)$ containing $X \in 2^{X}$. Then $W=2^{X}$.

Proof. If $F \in 2^{X}$ then $F \subseteq X$ and so $X \in \operatorname{cl}_{\sigma}\{F\}$, i.e. $F \in W$.

From Corollary 3.3. we immediately obtain

Corollary 3.4. $\left(2^{X}, \sigma\right)$ is always compact.

Corollary 3.5. $\left(2^{X}, \sigma\right)$ satisfies condition $(P 3)$

- Proof. Let $B_{1}, B_{2} \subseteq 2^{X}$ be nonempty and closed in $\left(2^{X}, \sigma\right)$. Suppose that $B_{1} \cap$ $B_{2}=\varnothing$. Then $2^{X}=\left(2^{X}-B_{1}\right) \cup\left(2^{X}-B_{2}\right)$ and we assume w.l.o.g. that $X \in 2^{X}-B_{1}$. By Corollary 3.3. we have $2^{X}-B_{1}=2^{X}$, i.e. $B_{1}=\varnothing$ which is a contradiction. So $B_{1} \cap B_{2} \neq=\varnothing$ and hence $\left(2^{X}, \sigma\right)$ satisfies (P3).

As a consequence, $\left(2^{X}, \sigma\right)$ is not $T_{1}$ whenever $\left|2^{X}\right| \geq 2$. 
In concluding this section we will consider the map $f:(X, \tau) \rightarrow\left(2^{X}, \sigma\right)$ where $f(x)=\operatorname{cl}_{\tau}\{x\}$ for each $x \in X$. It has been pointed out in [1] that $f$ is a dense embedding provided that $(X, \tau)$ is $T_{1}$. Our final result shows that the converse also holds.

Theorem 3.6. If $f:(X, \tau) \rightarrow\left(2^{X}, \sigma\right)$ where $f(x)=c l_{\tau}\{x\}$ for each $x \in X$ is an embedding then $(X, \tau)$ is $T_{1}$.

Proof. Suppose that $x, y \in X$ with $x \neq y$ and $y \in \operatorname{cl}_{\tau}\{x\}$. Since $f$ is one-to-one, $(X, \tau)$ is $T_{0}$ and so there exists $U \subseteq X$ open in $(X, \tau)$ with $x \in U$ and $y \notin U$. Since $f(U)$ is open in $f(X)$ there exists $V \subseteq X$ open in $(X, \tau)$ with $f(x) \in V^{*} \cap f(X) \subseteq f(U)$. Hence $f(y) \in f(U)$ since $\operatorname{cl}_{\tau}\{y\} \subseteq \operatorname{cl}_{\tau}\{x\}$. As $f$ is one-to-one, we have $y \in U$, a contradiction. This proves the $(X, \tau)$ is $T_{1}$.

Remark 3.7. The pair $\left(\left(2^{X}, \sigma\right), f\right)$ is a compactification of $(X, \tau)$ if and only if $(X, \tau)$ is a $T_{1}$ space.

\section{References}

[1] M. E. Abd El-Monsef, A. M. Kozae and A. A. Abo-Khadra, "Extensions generated by closed sets," Tamkang J. Math., 24 (1993), 189-193

[2] N. Bourbaki, General Topology, Part 1, Addison-Wesley, Reading, Mass. 1966.

[3] E. Michael, "Topologies on spaces of subsets," Trans. Amer. Math. Soc., 71 (1951), 152-182

[4] H. J. Schmidt, "Hyperspaces of quotient and subspaces I." Hausdorff topological spaces, Math Nachr., 104. (1981), 271-280 